\title{
Investigation on the Effects of Magnetic Saturation in Induction Machines During Transients
}

\author{
Alexander Conradi, Christoph Schmülling, Stefan Schmülling \\ Chair of Electrical Drives and Mechatronics \\ TU Dortmund University \\ 44227 Dortmund, Germany \\ alexander.conradi-,christoph.schmuelling-,stefan.schmuelling@tu-dortmund.de
}

\begin{abstract}
Magnetic saturation in electrical machines is of major influence on the operating characteristics during transients. For induction machines, the assumption of linear magnetic behavior of the iron core in classical machine models may be adequate for the calculation of steady-state rated operation. In operating points or during transients following a short-circuit or out-of-phase synchronization, where currents can reach values as high as $25 \mathrm{pu}$, especially the magnetic paths of the leakage fluxes are highly saturated, leading to a significant raise in currents and machine torque. Therefore an investigation on the effect of magnetic saturation has been performed by comparing inductances calculated by analytical methods for assumed linear magnetic behavior with results of static finite-element-method (FEM) models taking magnetic saturation into account. These results are subsequently converted into simplified current-depending parameter functions that can be used in transient machine models. Therefore, based on an enhanced space phasor approach, a newly modified machine model is presented, where the parameters can be used according to the dependencies worked out in the paper in order to identify deviations with the classical approach using constant inductances. Furthermore the newly acquired set of current-depending machine inductances is validated by simulating a series of transients using dynamic FEM calculation, showing a significant improvement in accuracy compared to sets of constant parameters.
\end{abstract}

Keywords - induction machines; machine inductances; magnetic saturation; FEM; transient simulation

\section{INTRODUCTION}

In induction machine models, magnetic flux linking the machine coils is commonly described by defining inductances for main and leakage flux paths and therefore modeling fluxes as functions of machine currents.

As the main inductance for reasons of efficiency is necessarily intended to be large compared to leakage inductances on constructing a machine, mathematical machine models are much more sensitive to variations in the leakage inductances than to the main inductance.

Knowledge of the leakage inductances of electrical machines is a decisive factor for calculating their operating performance, with starting torque and current, power factor and behavior at short circuit strongly depending on it in particular [3][4]. There are six principal leakage paths in induction machines, with stator and rotor leakage as well as differential leakage (zigzag and belt leakage) depending on the geometric cross section and the magnetic characteristics of the active iron part of the machine, while the remaining two (end-winding leakage and skew leakage) are determined by the bar skew along the $\mathrm{z}$-axis and the geometry of the winding parts (stator) and the short-circuit ring (rotor) outside the core region [1][2].

The total leakage inductance of an inductance motor is much smaller at standstill compared to operation at full-load or pullout torque. This is partly due to the reduction of the slot inductance in the rotor bars as a result of the skin effect at higher frequencies of the rotor current. Another important factor is the magnetic saturation of the material affecting only those inductances describing magnetic flux within the iron core. Especially in the case of machine transients under fault conditions (e.g. three or two phase short-circuits) or following out-of-phase synchronization with machine currents sometimes exceeding $20 \mathrm{pu}$, the reduction in the magnetic permeances as a result of occurring saturation is the major factor for reduced leakage inductances leading to higher currents.

The only significant influence of saturation of the main inductance is at no-load operation, where the main inductance directly determines the no-load current. For calculation of transient behavior or under load conditions at steady state, the influence is comparatively small.

The objective of the paper at hand is to investigate the core saturation and resulting inductance characteristics for variation of the main electrical operating parameters being the magnitude of stator and rotor current and their resulting phase angle. In induction machines with short-circuit-rotor, stator and rotor currents are strongly dependent, so an arbitrary variation of the three parameters may lead to investigation of combinations not occurring in real operation. Nevertheless, in order to derive consistent functional dependencies comprising all relevant operating points and in order to better understand the relevant circumstances determining magnetic saturation in induction machines, a full-variation approach is used to generate data which can be later reduced to functional dependencies covering subsets of effective operation points.

The results are compared with analytical approaches, where only magnetic voltage drops across the air gap and the machine slots are considered based on the assumption that permeability 
in these parts is small compared to the machine iron, hence magnetic voltage drops in the iron is negligible.

In order to show the impact of consideration of iron saturation in machine models, further investigations are made by comparing transient operations of the machine for parameters with and without functional dependencies on the machine currents by using an mathematical model based on a system of differential equations which is numerically solved, similar to the models described in [5] and [6] but newly enhanced for consideration of leakage path saturation.

The transient calculations are validated by comparison of the results against simulations performed with a dynamic FEM using the same cross section as for the static determination of the saturation characteristics.

\section{INVESTIGATION APPROACH}

\section{A. Machine under investigation}

The investigation has been carried out on a representative motor used for industrial purposes with apparent power around 1 MW. The machine iron has a typical magnetization characteristic as depicted in Figure 1.

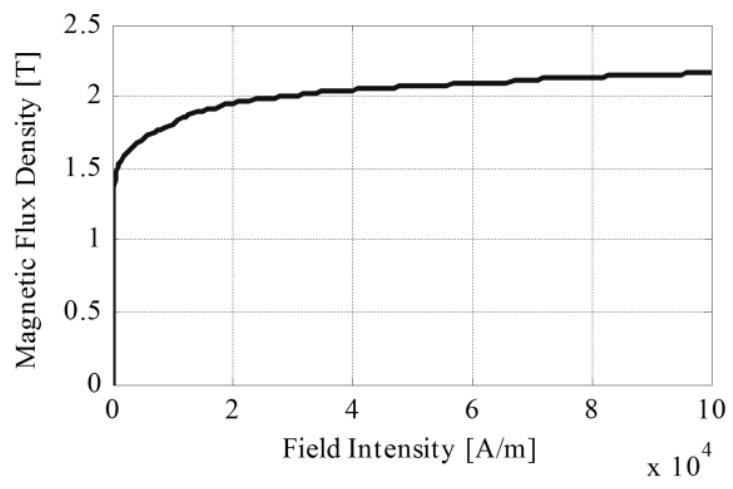

Figure 1: Magnetization curve

\section{B. Definition of Machine Inductances}

In common analytical induction machine models, the magnetic flux is split into the main flux linked to both stator and rotor windings, therefore being represented by the main inductance $L_{\mathrm{m}}$ and the leakage fluxes of stator and rotor represented by the inductances $L_{1 \sigma}$ and $L_{2 \sigma}^{\prime}$ in the T-equivalent circuit of Figure 2.

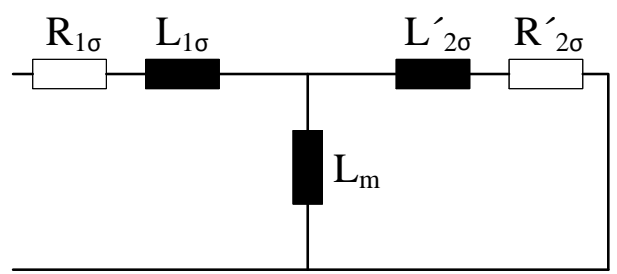

Figure 2: T-equivalent circuit of induction machines

By describing the machine with a complex-valued two-axis model [8], the three-phase (stator) or multi-phase (rotor cage) currents are represented by single phasors $\underline{i}_{1}=i_{1} \cdot e^{j \varphi_{1}}$ and $\underline{i}_{2}^{\prime}=i_{2}^{\prime} \cdot e^{j \varphi_{2}}$ which have a constant length $i_{1}$ and $i_{2}^{\prime}$ and rotate with constant speed at steady state operation with a therefore constant differential angle $\varphi$, which is generally defined by

$$
\varphi=\varphi_{1}-\varphi_{2}
$$
by

The flux linkages in the complex two axis model are defined

$$
\begin{aligned}
& \underline{\Psi}_{\mathrm{m}}=L_{\mathrm{m}} \cdot\left(\underline{i}_{1}+\underline{i}_{2}^{\prime}\right)=L_{\mathrm{m}} \cdot \underline{i}_{\mathrm{m}} \\
& \underline{\Psi}_{1 \sigma}=L_{1 \sigma} \cdot \underline{i}_{1}, \quad \underline{\Psi}_{2 \sigma}=L_{2 \sigma} \cdot \underline{i}_{2}
\end{aligned}
$$

with the exact same inductances as in the T-equivalent circuit model.

\section{Analytical Calculation of Machine Inductances}

For the assumption of homogenous linear magnetic characteristics of the material with $\mu_{\mathrm{r}} \gg>1$ and therefore normal magnetic field lines across the airgap and the slots the machine inductances can be calculated analytically.

The machine's main inductance can be calculated by:

$$
L_{\mathrm{m}}=2 \cdot \mu_{0} \cdot \frac{l_{\mathrm{z}} \cdot \tau_{\mathrm{p}}}{\delta \cdot p} \cdot m \cdot\left(N_{1} \cdot k_{w 1}\right)^{2}
$$

By assuming a Dirichlet boundary condition (tangential magnetic field lines) at the airgap and normal magnetic field lines across the slots for the leakage flux, the machine's leakage inductances can be calculated by only taking the geometry into account of the slots, the connection of the single coils and the winding number.

For stators with two-layer windings, the linked flux on a slot has to be divided by origin and destination according to Figure 3.

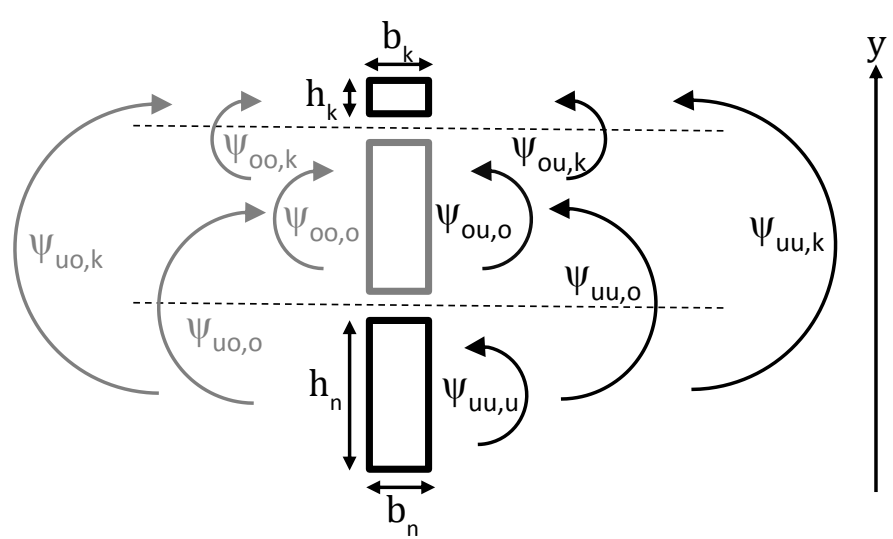

Figure 3: Linked stator leakage flux in a two-layer winding 


$$
\begin{aligned}
& L_{\mathrm{uu}, \mathrm{u}}=L_{\mathrm{oo}, \mathrm{o}}=\frac{1}{3} \mu_{0} \cdot N^{2} \cdot \frac{h_{\mathrm{n}}}{b_{\mathrm{n}}} \cdot l_{\mathrm{z}} \\
& L_{\mathrm{uu}, \mathrm{o}}=\mu_{0} \cdot N^{2} \cdot \frac{h_{\mathrm{n}}}{b_{\mathrm{n}}} \cdot l_{\mathrm{z}} \\
& L_{\mathrm{uo}, \mathrm{o}}=\frac{1}{2} \mu_{0} \cdot N^{2} \cdot \frac{h_{\mathrm{n}}}{b_{\mathrm{n}}} \cdot l_{\mathrm{z}} \\
& L_{\mathrm{ou}, \mathrm{o}}=\frac{1}{2} \mu_{0} \cdot N^{2} \cdot \frac{h_{\mathrm{n}}}{b_{\mathrm{n}}} \cdot l_{\mathrm{z}} \\
& L_{\mathrm{k}}=L_{\mathrm{uu}, \mathrm{k}}=L_{\mathrm{uo}, \mathrm{k}}=L_{\mathrm{oo}, \mathrm{k}}=L_{\mathrm{ou}, \mathrm{k}}=\mu_{\mathrm{r}, \mathrm{k}} \cdot \mu_{0} \cdot N^{2} \cdot \frac{h_{\mathrm{k}}}{b_{\mathrm{k}}} \cdot l_{\mathrm{z}},
\end{aligned}
$$

For rectangular slots o and $\mathrm{u}$ topped by a rectangular slot wedge as depicted in Figure 3, the inductances can be calculated by using Ampere's law with (5), the total linked flux in the coils of upper and lower winding layer can be expressed in terms of the coil currents by

$$
\begin{aligned}
& \psi_{\mathrm{u}}=\underbrace{\left(L_{\mathrm{uu}, \mathrm{u}}+L_{\mathrm{uu}, \mathrm{o}}+L_{\mathrm{k}}\right)}_{L_{\mathrm{uu}}} \cdot i_{\mathrm{u}}+\underbrace{\left(L_{\mathrm{ou}, \mathrm{o}}+L_{\mathrm{ou}, \mathrm{k}}\right)}_{L_{\mathrm{ou}}} \cdot i_{\mathrm{o}} \\
& \psi_{\mathrm{o}}=\underbrace{\left(L_{\mathrm{oo}, \mathrm{o}}+L_{\mathrm{oo}, \mathrm{k}}\right)}_{L_{\mathrm{oo}}} \cdot i_{\mathrm{o}}+\underbrace{\left(L_{\mathrm{uo}, \mathrm{o}}+L_{\mathrm{uo}, \mathrm{k}}\right)}_{L_{\mathrm{uo}}} \cdot i_{\mathrm{u}} .
\end{aligned}
$$

A standard three-phase two-layer pitched winding as depicted in Figure 4 is described by its coil span $C$, slots per phase $N$, windings per phase $w$, the number of pole pairs $p$ and the number of parallel coils per phase $Q$. The $N$ slots per phase can be divided into $N_{1}$ slots holding two coils of the same phase and $N_{2}$ slots holding two coils of different phases, so that

$$
N=N_{1}+N_{2} \text {. }
$$

$N_{1}$ is given by

$$
N_{1}=2 \mathrm{p} \cdot C-2 \cdot N
$$

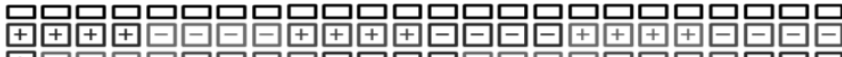

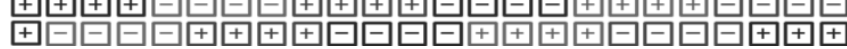

Figure 4: Schematic two-layer winding

With these information, the coupled flux for all phases can be calculated as

$$
\begin{aligned}
\psi_{a}= & \underbrace{\frac{1}{Q^{2}}\left(\frac{N}{3} \cdot\left(L_{\mathrm{uu}}+L_{\mathrm{oo}}\right)+N_{1} \cdot\left(L_{\mathrm{ou}}+L_{\mathrm{uo}}\right)\right)}_{L_{\mathrm{ss}}} \cdot i_{\mathrm{a}} \\
& -\underbrace{\frac{1}{Q^{2}} N_{2} \cdot L_{\mathrm{ou}}}_{L_{\mathrm{ou}, \text { eff }}} \cdot i_{\mathrm{b}}-\underbrace{\frac{1}{Q^{2}} N_{2} \cdot L_{\mathrm{uo}}}_{L_{\mathrm{uo}, \text { eff }}} \cdot i_{\mathrm{c}} \\
\psi_{\mathrm{b}}= & L_{\mathrm{ss}} \cdot i_{\mathrm{b}}-\left(L_{\mathrm{ou}, \mathrm{eff}} \cdot i_{\mathrm{c}}+L_{\mathrm{uo}, \mathrm{eff}} \cdot i_{\mathrm{a}}\right) \\
\psi_{\mathrm{c}}= & L_{\mathrm{ss}} \cdot i_{\mathrm{c}}-\left(L_{\mathrm{ou}, e f f} \cdot i_{\mathrm{a}}+L_{\mathrm{uo}, \mathrm{eff}} \cdot i_{\mathrm{b}}\right) .
\end{aligned}
$$

With knowledge of $L_{\mathrm{ss}}, L_{\mathrm{ou}, \mathrm{eff}}$ and $L_{\mathrm{uo}, \mathrm{eff}}$, and in the case of $L_{\text {ou,eff }}=L_{\text {uo,eff }}$ the total slot leakage inductance $L_{1 \sigma, \text { slot }}$ can be finally calculated by

$$
L_{1 \sigma, \mathrm{slot}}=L_{\mathrm{ss}}+L_{\mathrm{ou}, \mathrm{eff}} .
$$

Other than the stator winding the rotor bars of an induction machine are designed as solid conductors. Therefore the skin effect has to be taken into regard for a proper calculation of the rotor slot leakage inductance. Additionally to the calculation of the bar inductance similar to the method depicted in Figure 3, a frequency-dependent factor $k_{i}$ must be multiplied with the DCinductance of a rotor bar:

$$
L_{r}=L_{r, D C} \cdot k_{i}(f)=\frac{1}{3} \mu_{0} \cdot \frac{h_{\mathrm{n}}}{b_{\mathrm{n}}} \cdot l_{\mathrm{z}} \cdot k_{i}(f)
$$

with

$$
k_{i}(f)=\frac{3}{2 h_{l}^{\prime}} \frac{\sinh \left(2 h_{l}^{\prime}\right)-\sin \left(2 h_{l}^{\prime}\right)}{\cosh \left(2 h_{l}^{\prime}\right)-\cos \left(2 h_{l}^{\prime}\right)}
$$

and

$$
h_{l}^{\prime}=\sqrt{\pi \cdot f \cdot \mu_{0} \cdot \gamma} \cdot h_{l} .
$$

Still, equation (11) is only valid, when the rotor bars are of rectangular shape, which will suit as a good approximation in most cases. For better consideration of the exact shape, there have been methods proposed, dividing the rotor bar in different sections and therefore approximating the slot shape more precisely [7].

\section{FEM-Calculation of Machine Inductances}

In order to take the effect of iron saturation into account, it is necessary to define these inductances as functions of the three parameters describing the machine's current linkage:

$$
L_{\mathrm{m}}\left(\underline{i}_{1}, \underline{i}_{2}^{\prime}, \varphi\right), L_{1 \sigma}\left(\underline{i}_{1}, \underline{i}_{2}^{\prime}, \varphi\right), L_{2 \sigma}\left(\underline{i}_{1}, \underline{i}_{2}^{\prime}, \varphi\right)
$$

All fluxes subject to saturation effects (i.e. main flux and leakage flux within the iron core) can be modeled on a geometric 2D cross-section of the machine with known magnetic characteristics (non-linear magnetization curve) of the material and a given current linkage in stator and rotor windings (represented by current magnitude of stator and rotor current and phase angle). With these information, the analysis for each combination of the electric parameters forming a specific current linkage is performed in three steps.

1. The machine model is fed with both stator and rotor current linkage. The resulting permeability of the material on the cross section of the machine is stored.

2. The same permeability as in 1 . is assumed to be independent of the resulting flux, hence a linear model with the same state of saturation as in 1 is set up. The machine is then fed only with stator current, so $\underline{i}_{\mathrm{m}}=\underline{i}_{1}$. The resulting flux linkage with the rotor gives the main inductance for the operating point $\left(i_{1}, i_{2}, \varphi\right)$ with

$$
L_{\mathrm{m}}=\frac{\underline{\Psi}_{\mathrm{m}}}{\underline{i}_{\mathrm{m}}}=\frac{\Psi_{\mathrm{m}}}{i_{\mathrm{m}}}=\frac{\Psi_{1,2}^{\prime}}{i_{1}}=\frac{\Psi_{2}^{\prime}}{i_{1}} .
$$


As a result of the previously calculated permeability, step 2. can be equally processed by only feeding the rotor and evaluation of the stator flux giving

$$
L_{\mathrm{m}}=\frac{\underline{\Psi}_{\mathrm{m}}}{\underline{i}_{\mathrm{m}}}=\frac{\Psi_{\mathrm{m}}}{i_{\mathrm{m}}}=\frac{\Psi_{2,1}}{\underline{i}_{2}^{\prime}}=\frac{\Psi_{1}}{\underline{i}_{2}^{\prime}} .
$$

3. The Dirichlet boundary condition is assigned to the circumference along the middle of the air gap, while the magnetic permeability along the cross section is the same as in 2. All fluxes from the model with set Dirichlet boundary are denoted as $\Psi^{\mathrm{D}}$. As a result, all the flux linked with a winding in either stator or rotor is only dependent on the winding's own current and can be interpreted as leakage flux. This is due to the fact, that the total flux linkage $\Psi_{1,1}$ or $\Psi_{2,2}$ is by approximation reduced by the coupling flux $\Psi_{1,2}$ and $\Psi_{2,1}$ respectively by setting the Dirichlet boundary condition, so that

$$
\begin{aligned}
& \Psi_{1}^{\mathrm{D}}=\Psi_{1,1}^{\mathrm{D}}=\Psi_{1,1}-\Psi_{2,1}=\Psi_{1, \sigma} \\
& \Psi_{2}^{\mathrm{D}}=\Psi_{2,2}^{\mathrm{D}}=\Psi_{2,2}-\Psi_{1,2}=\Psi_{2, \sigma} .
\end{aligned}
$$

The leakage inductances for the operating point $\left(i_{1}, i_{2}, \varphi\right)$ are then calculated according to (3) by

$$
\begin{aligned}
& L_{1 \sigma}=\frac{\underline{\Psi}_{1 \sigma}}{\underline{i}_{1}}=\frac{\Psi_{1 \sigma}}{i_{1}}=\frac{\Psi_{1}^{\mathrm{D}}}{i_{1}} \\
& L_{2 \sigma}=\frac{\underline{\Psi}_{2 \sigma}}{\underline{i}_{2}}=\frac{\Psi_{2 \sigma}}{i_{2}}=\frac{\Psi_{2}^{\mathrm{D}}}{i_{2}} .
\end{aligned}
$$

\section{E. Enhancement of Dynamic Machine Equations for \\ Consideration of Saturation Effects}

In order to include the saturated machine inductances calculated with the method presented into the complex space phasor model a few adjustment have to be made. As will be shown in the results section, the functional dependencies for the induction machine at operation can approximately be formulated as

$$
L_{\mathrm{m}}\left(\underline{i}_{\mathrm{m}}\right) \quad L_{1 \sigma}\left(\underline{i}_{1}\right) \quad L_{2 \sigma}^{\prime}\left(\underline{i}_{2}^{\prime}\right) .
$$

Tang [6] has presented a dynamic mathematical model with consideration of saturation effects in the main flux path by introducing a differential inductance $L_{\mu \mathrm{d}}$, giving

$$
\frac{\mathrm{d} \underline{\psi}_{\mu}}{\mathrm{d} t}=L_{\mu} \frac{\mathrm{d} \underline{i}_{\mu}}{\mathrm{d} t}+\underline{i}_{\mu} \frac{\mathrm{d} L_{\mu}}{\mathrm{d} i_{\mu}} \frac{\mathrm{d} i_{\mu}}{\mathrm{d} t}=L_{\mu} \frac{\underline{i}_{\mu}}{\mathrm{d} t}+\underline{i}_{\mu} \frac{L_{\mu \mathrm{d}}-L_{\mu}}{i_{\mu}} \frac{\mathrm{d} i_{\mu}}{\mathrm{d} t}
$$

for the time derivative of the main flux $\underline{\psi}_{\mu}$. At this stage, a problem occurs as the absolute value of the magnetizing current $i_{\mu}$ is not a system variable, that can be solved for. Still, a workaround can be found by introducing the complexconjugated machine currents $\underline{i}_{1}^{*}$ and $\underline{i}_{2}^{\prime *}$ as new system variables and enhancing the system of differential equations for the complex-conjugated counterpart.
The resulting system bears strong resemblance to the method of symmetrical components, with the original set of variables and the complex-conjugates rotating in opposite directions. By writing the magnetizing current using Euler's formula

$$
\underline{i}_{\mu}=i_{\mu} e^{j \xi_{\mu}} \quad \underline{i}_{\mu}^{*}=i_{\mu} e^{-j \xi_{\mu}}
$$

after a few rearrangements (20) can be formulated as

$$
\frac{\mathrm{d} \underline{\psi}_{\mu}}{\mathrm{d} t}=L_{\mu, \mathrm{a}} \frac{\mathrm{d} \underline{i}_{\mu}}{\mathrm{d} t}+L_{\mu, \mathrm{b}} e^{j 2 \xi_{\mu}} \frac{\mathrm{d} \underline{i}_{\mu}^{*}}{\mathrm{~d} t}
$$

with

$$
L_{\mu, \mathrm{a}}=\frac{L_{\mu \mathrm{d}}+L_{\mu}}{2} \quad L_{\mu, \mathrm{b}}=\frac{L_{\mu \mathrm{d}}-L_{\mu}}{2} .
$$

The two original equations are then given by

$$
\begin{aligned}
& \underline{u}_{1}=R_{\mathrm{s}} \underline{i}_{1}+L_{\sigma \mathrm{s}} \frac{\mathrm{d} \underline{i}_{1}}{\mathrm{~d} t}+L_{\mu, \mathrm{a}} \frac{\mathrm{d} \underline{i}_{\mu}}{\mathrm{d} t}+L_{\mu, \mathrm{b}} e^{j 2 \xi_{\mu}} \frac{\mathrm{d} \underline{i}_{\mu}^{*}}{\mathrm{~d} t} \\
& 0=R_{\mathrm{r}}^{\prime} \underline{\underline{\prime}}_{2}^{\prime}+L_{\sigma \mathrm{r}}^{\prime} \frac{\mathrm{d} \underline{i}_{2}^{\prime}}{\mathrm{d} t}+L_{\mu, \mathrm{a}} \frac{\mathrm{d} \underline{i}_{\mu}}{\mathrm{d} t}+\ldots \\
& \ldots+L_{\mu, \mathrm{b}} e^{j 2 \xi_{\mu}} \frac{\mathrm{d} \underline{i}_{\mu}^{*}}{\mathrm{~d} t}-j \omega_{\mathrm{el}}\left(L_{\sigma \mathrm{r}}^{\prime} \underline{i}_{2}^{\prime}+L_{\mu} \underline{i}_{\mu}\right)
\end{aligned}
$$

and must be complemented by their complex-conjugated counterparts.

The model of Tang can be enhanced for consideration of magnetic saturation in the leakage flux paths of the stator and rotor of the machine, so that all dependencies given by (19) can be used for transient calculation. Again using Euler's formula, the complex-conjugates of the original currents are written as

$$
\underline{i}_{1}^{*}=i_{1} e^{-j \xi_{1}} \quad \underline{i}_{2}^{\prime *}=i_{2} e^{-j \xi_{2}} .
$$

By defining

$$
\begin{array}{ll}
L_{\sigma \mathrm{s}, \mathrm{a}}=\frac{L_{\sigma \mathrm{sd}}+L_{\sigma \mathrm{s}}}{2} & L_{\sigma \mathrm{s}, \mathrm{b}}=\frac{L_{\sigma \mathrm{sd}}-L_{\sigma \mathrm{s}}}{2} \\
L_{\sigma \mathrm{r}, \mathrm{a}}^{\prime}=\frac{L_{\sigma \mathrm{d}}^{\prime}+L_{\sigma \mathrm{r}}^{\prime}}{2} & L_{\sigma \mathrm{r}, \mathrm{b}}^{\prime}=\frac{L_{\sigma \mathrm{rd}}^{\prime}-L_{\sigma \mathrm{r}}^{\prime}}{2}
\end{array}
$$

similarily to (23) with

$$
L_{\sigma \mathrm{sd}}=\frac{\mathrm{d} \psi_{\sigma 1}}{\mathrm{~d} i_{1}} \quad L_{\sigma \mathrm{dd}}^{\prime}=\frac{\mathrm{d} \psi_{\sigma 2}^{\prime}}{\mathrm{d} i_{2}}
$$

representing the time derivatives of the leakage flux of stator and rotor for the absolute values of the currents, the complete system of differential equations is given by 


$$
\begin{aligned}
& {\left[\begin{array}{c}
\underline{u}_{1} \\
\underline{u}_{1}^{*} \\
0 \\
0
\end{array}\right]=\left[\begin{array}{cccc}
R_{\mathrm{s}} & 0 & 0 & 0 \\
0 & R_{\mathrm{s}} & 0 & 0 \\
0 & 0 & R_{\mathrm{r}}^{\prime} & 0 \\
0 & 0 & 0 & R_{\mathrm{r}}^{\prime}
\end{array}\right]\left[\begin{array}{c}
\underline{i}_{1} \\
\underline{i}_{1}^{*} \\
\underline{i}_{2}^{\prime} \\
\underline{i}_{2}^{\prime *}
\end{array}\right]} \\
& -j \omega_{\mathrm{el}}\left[\begin{array}{cccc}
0 & 0 & 0 & 0 \\
0 & 0 & 0 & 0 \\
L_{\mu} & 0 & L_{\sigma \mathrm{r}}^{\prime}+L_{\mu} & 0 \\
0 & -L_{\mu} & 0 & -L_{\sigma \mathrm{r}}^{\prime}-L_{\mu}
\end{array}\right]\left[\begin{array}{c}
\underline{i}_{1} \\
\underline{i}_{1}^{*} \\
\underline{i}_{2}^{\prime} \\
\underline{i}_{2}^{\prime}
\end{array}\right]
\end{aligned}
$$

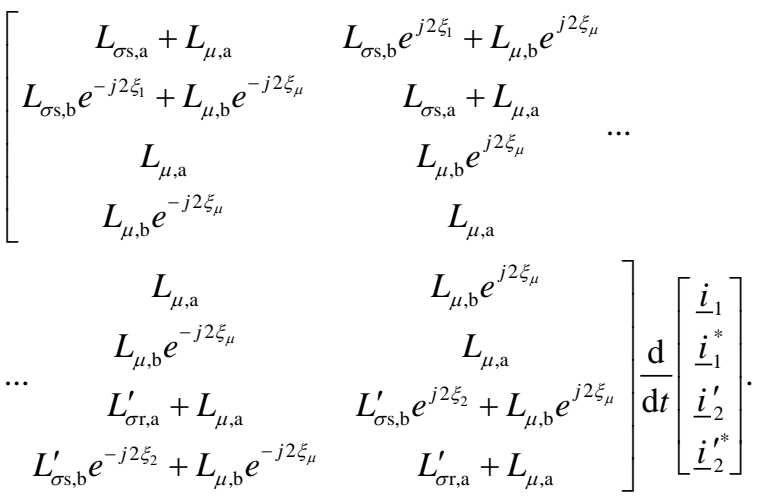

\section{F. Calculation of Machine Transients Using FEM}

In most induction machines, effects of magnetic saturation can only be seen in cases where machine currents reach multiples of $1 \mathrm{pu}$. Still, these operation sequences all exhibit different behavior in terms of frequency of rotor currents, transient torque, phase symmetry and interaction with the supply grid to be accounted for.

Therefore a selection of the transients covering the variety of possible operating characteristics has been chosen by simulation of the

- $\quad$ starting from standstill

- three-phase short circuit

- two-phase short circuit

- $\quad$ out of phase synchronization $\left(180^{\circ}\right.$ phase angle).

Simulations have been performed using Cedrat Flux (Cedrat S.A., Meylan Cedex, France), which is able to simulate static and transient behavior of electrical machines and any electromechanical setup in general.

\section{RESULTS}

FEM-Simulations have been carried out according to section II $D$. by varying the absolute value of stator and rotor current over a range from 0 to $20 \mathrm{pu}$ as well as the angle between the currents in five steps from $0^{\circ}$ up to $180^{\circ}$.

Figure 5 shows the collected database up to currents of 4 pu for calculation of the main inductance, where obviously the inductances for higher currents exhibit stronger saturation and therefore smaller values. Focusing on the diagonal with identical absolute values of stator and rotor currents, the phase angle is of major influence to the inductances, as for phase angles of $180^{\circ}$, the main flux paths are theoretically field-free, and only leakage flux remains, leading to high values for the main inductances even for high currents in stator and rotor.

For the case of one current being larger than the other or phase angles different from $180^{\circ}$, the values of the inductances decrease significantly.

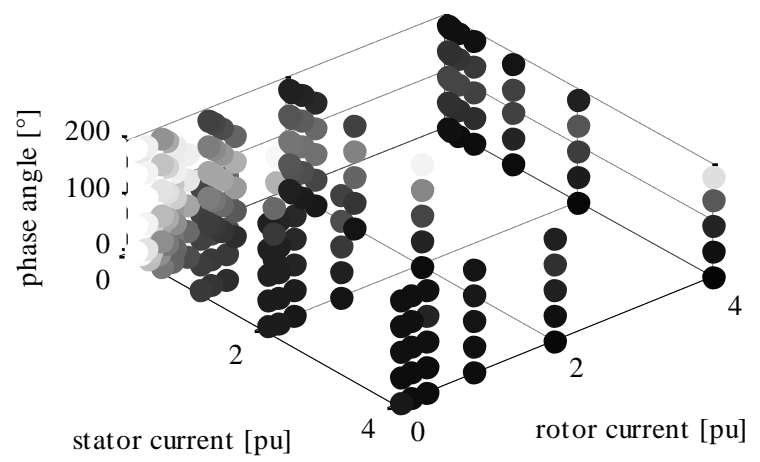

Figure 5: Main inductance resulting from variation of stator current, rotor current and phase angle

In order to closer examine certain phenomena, representative two-dimensional plots give a better focus. Figure 6 shows the main inductance for constant absolute stator current of 2 pu and variation of rotor current for phase angle of $0^{\circ}$ and $180^{\circ}$. For a phase angle of $0^{\circ}$, stator and rotor current add up to a common magnetizing current, hence the strictly monotonically decreasing inductance curve for rising rotor current. In contrast, the main inductance exhibits a maximum at 2 pu rotor current for a phase angle of $180^{\circ}$ as a result of the disappearing magnetizing current, with the main inductance close to $1 \mathrm{pu}$.

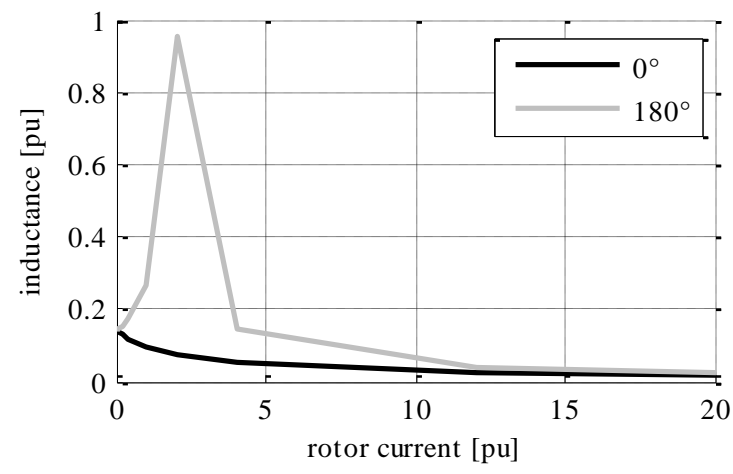

Figure 6: Main inductance for constant stator current of $2 \mathrm{pu}$

If the main inductance is considered to be dependent on the magnitude of the magnetizing current, as indicated by the results above, its functional description can be approximated by

$$
L_{\mathrm{m}}\left(\underline{i}_{1}, \underline{i}_{2}^{\prime}, \varphi\right)=L_{\mathrm{m}}\left(\underline{i}_{1}+\underline{i}_{2}^{\prime}\right)=L_{\mathrm{m}}\left(\underline{i}_{\mathrm{m}}\right),
$$

which can be derived from the simulations performed by simply varying stator current, where rotor currents remain 0 pu leading to the characteristic shown in Figure 7, where the constant value (grey) represents the analytically calculated main inductance. 


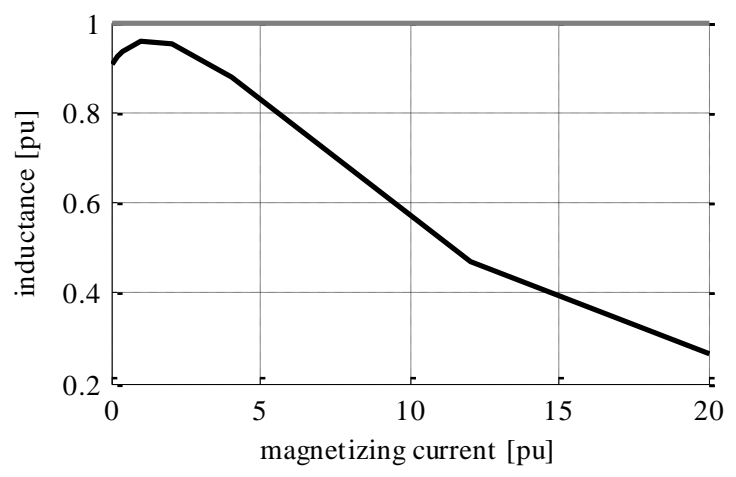

Figure 7: Comparison of saturated (black line) and non-saturated (grey line) main inductance

Calculation of the leakage inductances has been carried out for the same variation of current magnitudes and phase angle as the stator. Still, better insight is given by focusing on specific examples as given in Figure 8, where for constant stator current of $4 \mathrm{pu}$, the rotor current is varied for phase angles of $0^{\circ}$ and $180^{\circ}$ (dashed lines black and light grey) and vice versa, the stator current is varied for phase angles of $0^{\circ}$ and $180^{\circ}$ (solid lines black and light grey) with constant rotor current of $4 \mathrm{pu}$.

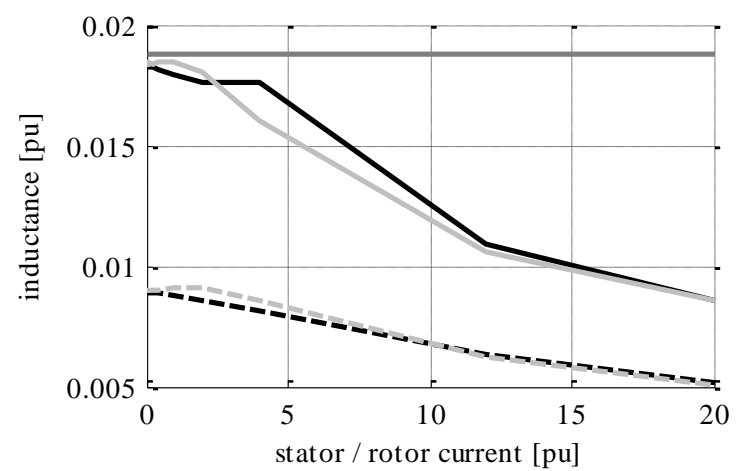

Figure 8: Stator (solid lines) and rotor (dashed lines) leakage inductance for phase angle of $0^{\circ}$ (back lines) and $180^{\circ}$ (light grey lines)

Apart from the basic dependency on the stator current for the stator leakage inductance and the rotor current for the rotor leakage inductance, there is an interesting difference between the curves. For the rotor leakage inductance, the value for $0^{\circ}$ phase angle is always smaller, meaning saturation of the flux path is stronger. For the stator leakage inductance, the value increases towards the value of $4 \mathrm{pu}$, interestingly for a phase angle of $0^{\circ}$, opposed to the phenomenon seen in Figure 6, where the main inductance reached its peak for equal current magnitudes and a phase angle of $180^{\circ}$.

The reason for this is that the part above the bars in the rotor of the machine under examination is of air, whereas the slot wedges in the stator are made of saturable material. This leads to leakage fluxes eliminating each other in the case of phase angle of $0^{\circ}$, making the leakage path across the stator slot wedges field-free for the case of identical current magnitudes, whereas the leakage path above the rotor bars is of the identical permeability of air all along and therefore not showing this phenomenon.
Due to the functional principle of the induction machine with induced rotor voltages driving currents in order to match the change in the exciting stator field, stator and rotor currents at operation are of similar magnitude, which means that the most intuitive approximation for analytical modeling of leakage path saturation would be the assumption, that

1. Only those combinations of stator and rotor currents where $\underline{i}_{1}=\underline{i}_{2}^{\prime}$ have to be considered.

2. Leakage inductances may be expressed as functions of only one current, giving simplified functions $L_{1 \sigma}\left(\underline{i}_{1}\right)$ and $L_{2 \sigma}\left(\underline{i}_{2}^{\prime}\right)$.

Figure 9 depicts the resulting inductance curves from the simulation data for the assumptions given above.

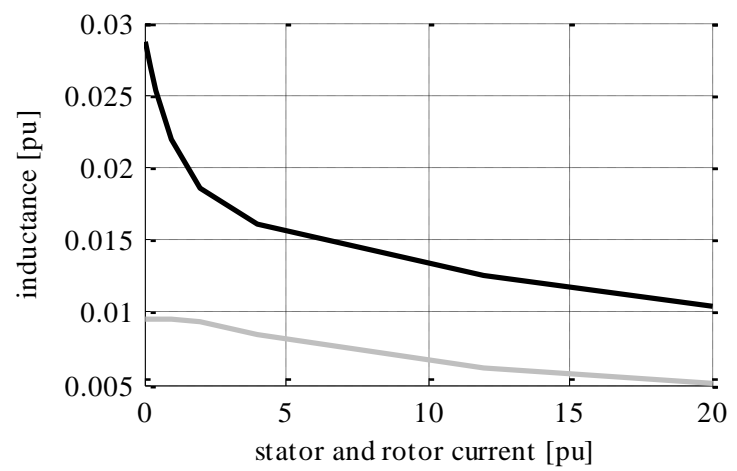

Figure 9: Stator (black line) and rotor (light grey line) leakage inductances for phase angle of $180^{\circ}$

By applying the induction functions depicted in Figure 7 and Figure 9 on the enhanced machine model given by (28), the transient behavior of the machine changes significantly compared to simulations made with the analytical dataset for assumed linear-magnetic behavior.

Any investigation on the effect of magnetic saturation on machine transients will eventually come down to the degree of precision that can be reached calculating the peak values of the torque, since it affects the mechanical design of the machine itself as well as the safety regards concerning coupled mechanical components.

Still, for a better understanding of the essential differences to dynamic machine modeling coming with consideration of magnetic saturation, looking at the coupled machine fluxes is most useful to begin with since its direct relation to the topic at investigation. The third important physical quantity examined is the machine current, being responsible for the excitation of the flux as well as representing a crucial quantity concerning safety regards.

Therefore Figures 10-14 show these three quantities (coupled flux and current of one phase, electrical torque) calculated by FEM and the transient machine model with and without consideration of magnetic saturation for the transients described in II. $F$.

Looking at the time transients in Figure 10, showing the machine startup from standstill, the most obvious differences 
between results of the different simulation methods can be observed in the coupled flux. The enhanced model (dark grey) shows good accordance with the FEM, where the coupled flux given by the model using constant inductances calculates for higher values throughout the entire starting process up until $t=0.6 \mathrm{~s}$ where currents reduce significantly.

The current itself shows according characteristics as higher coupled fluxes as a result of non-saturated inductances lead to lower currents for the model with constant parameters. The effect tends to show stronger for extreme currents as can be seen at the first peak, where the values of the model are at $81.5 \%$ (constant parameters - I) and $92.2 \%$ (consideration of saturation - II) of the FEM-simulation.

The effect on the transient torque is rather small looking at the entire startup. Still, looking at the important peak value at the beginning again gives a deviation of $13.4 \%$ (I) and only $2.2 \%$ (II) with FEM.
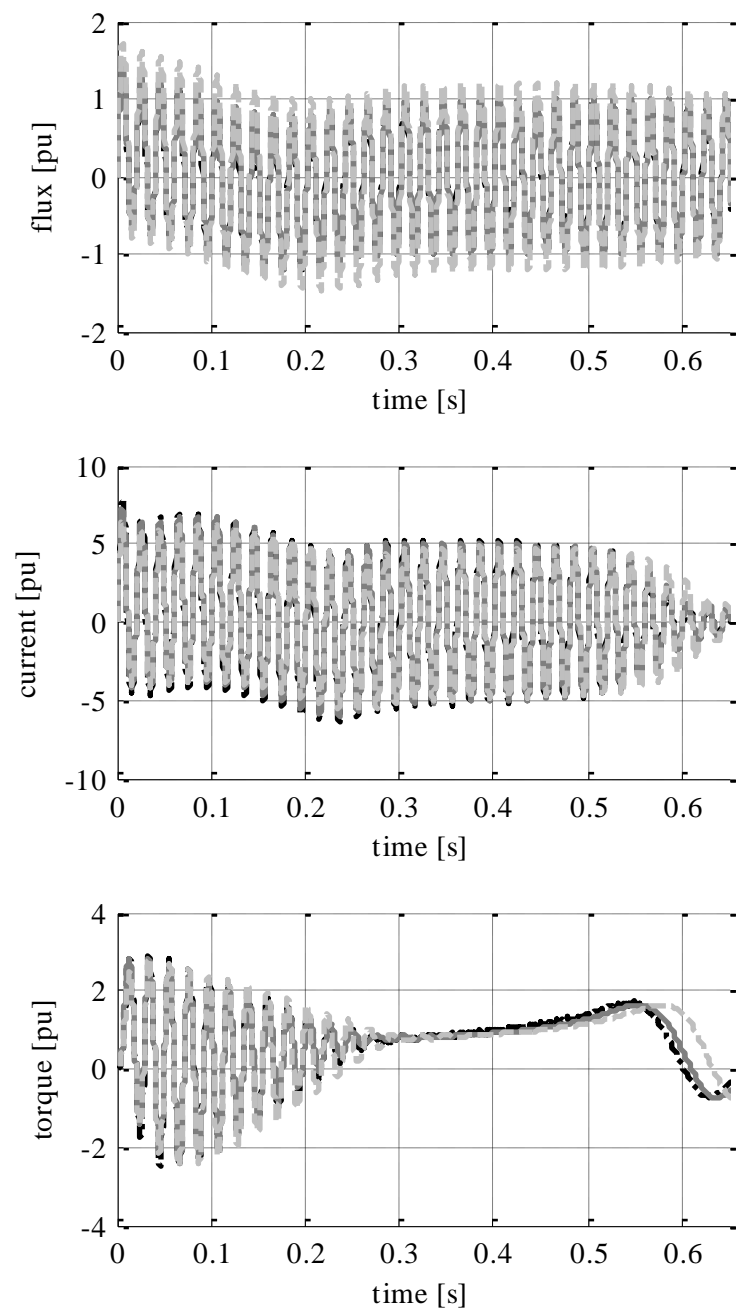

Figure 10: Transient behavior of flux, current and torque (black line-FEM, dark grey line -with saturation, light grey line -without saturation)

A similar conclusion can be drawn for the three-phase short circuit depicted in Figure 11. Again, the most significant differences show for the coupled flux, where using constant inductances in the model, leads to stronger oscillations compared to the smooth damping of the flux given by the FEM and the enhanced model. For the current this effect leads to a much improved accordance of the machine model with the FEM, where deviations at peak currents reduce from $14.8 \%$ (I) to $4.7 \%$ (II).

At peak value, the torque is also calculated significantly closer to FEM results (-5.8 pu) with -5.7 pu compared to $-5.1 \mathrm{pu}$ of the model using constant values.
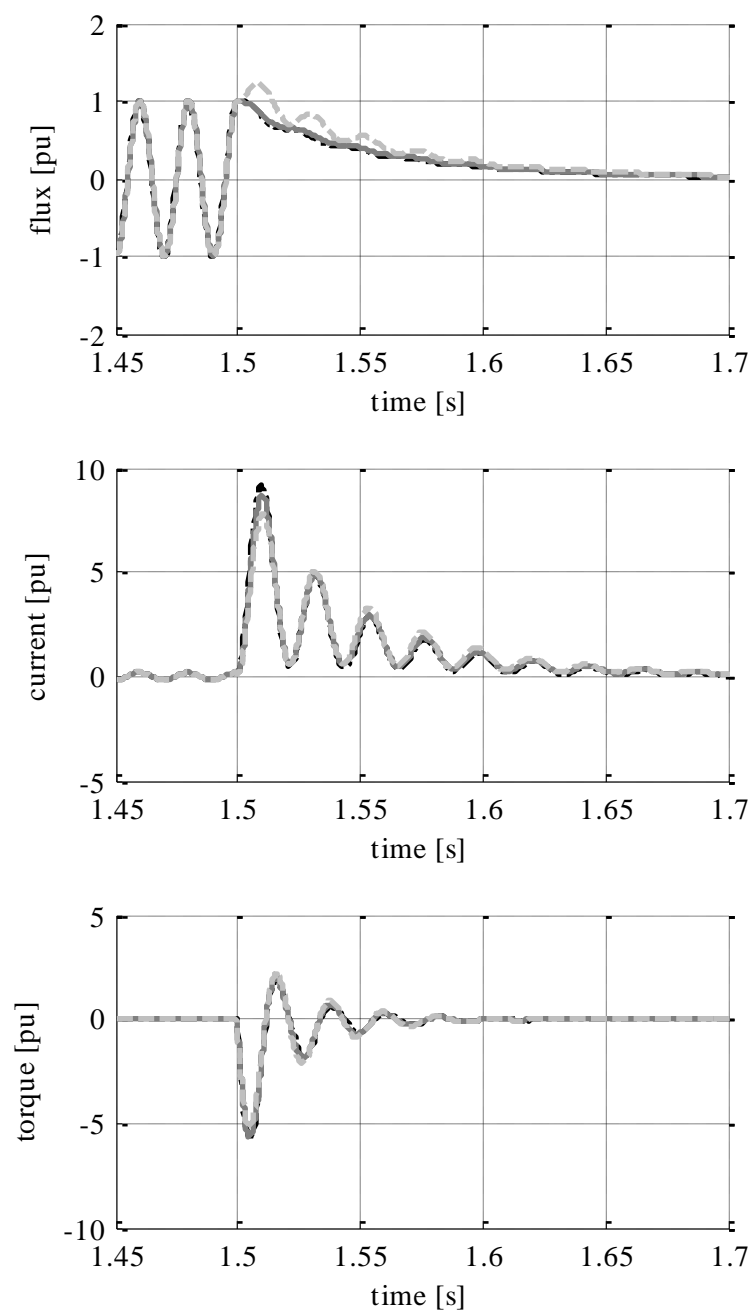

Figure 11: Three phase sudden short circuit (black line -FEM, dark grey linewith saturation, light grey line - without saturation)

From the transients discussed in this paper, the two-phase short circuit stands out as being of non-symmetric nature, as only the two short circuited phases (phase a and b) are leading current. Due to this circumstance, the effect of saturation mainly shows in the coupled flux of those phases as the self-linkage exceeds the flux coming from mutual linkage, so that the fluxpaths for the phase with zero current (phase c) are not getting into saturation mode, hence no substantial deviation is showing between the results of the models I and II (c.f. Figure 12). 

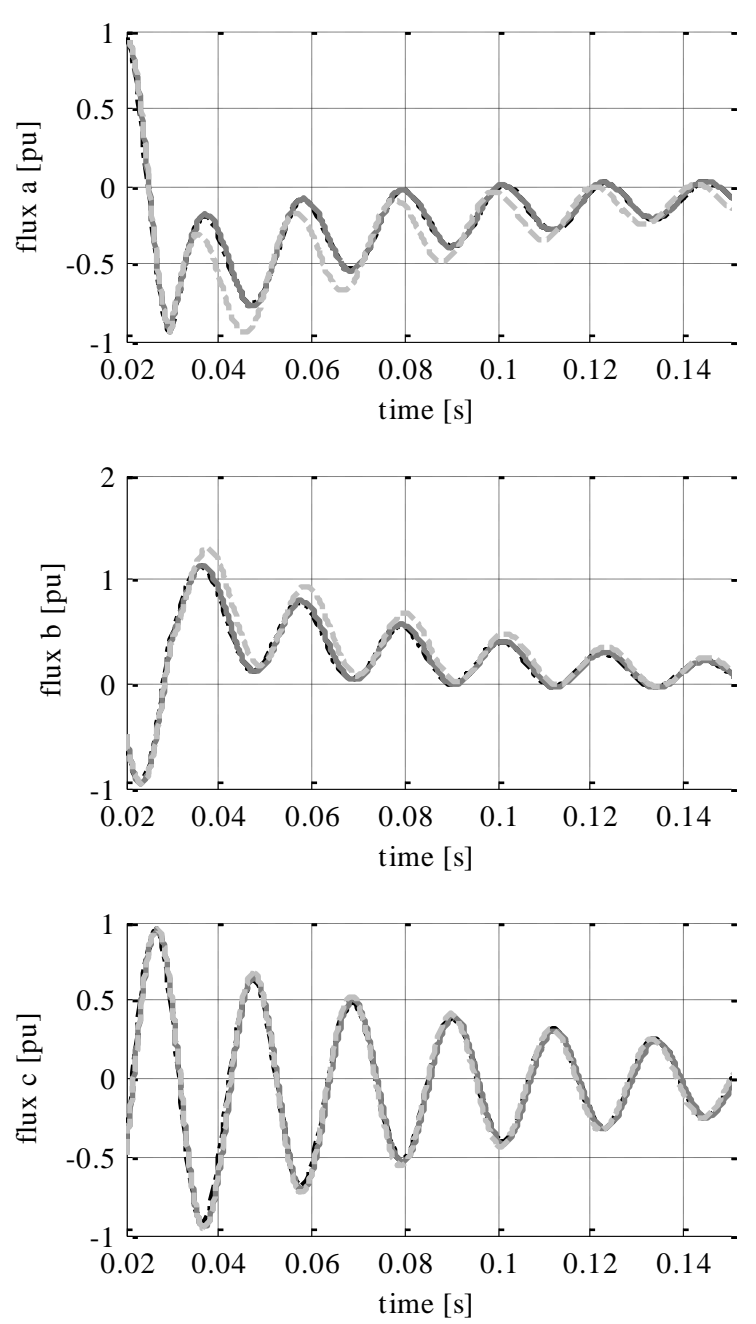

Figure 12: Two-phase short circuit behavior of fluxes (black line-FEM, dark grey line -with saturation, light grey line -without saturation)

The currents as well as the electric torque again show much improved accordance between the enhanced model (II) and the FEM by reducing the deviations for maximum peak of the current from $14.5 \%$ to $3.1 \%$ and for the torque from $12 \%$ to $2.4 \%$.

The out of phase synchronization with a $180^{\circ}$ phase angle can be found in literature [9] to be the induction machine transient producing the highest currents and given the strong dependence of ampere-turns to produced flux. It is not surprising that deviations between results of the enhanced model (II) and the basic model (I) become most obvious in this case.
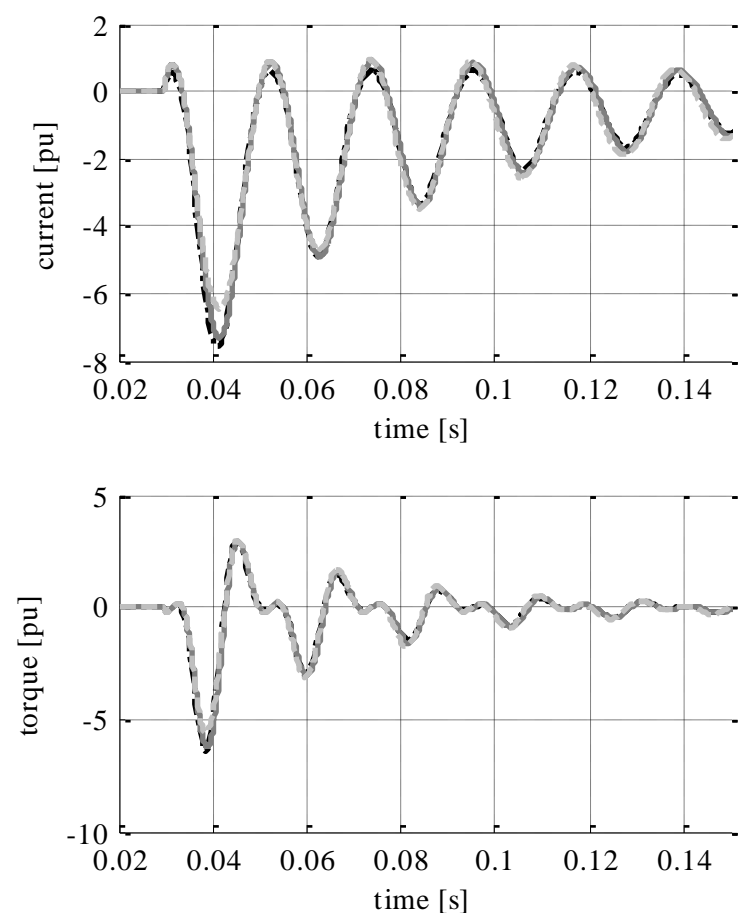

Figure 13: Two-phase short circuit behavior of current and torque (black line -FEM, dark grey line -with saturation, light grey line - without saturation)

For the machine investigated, the current reaches a peak value of $25 \mathrm{pu}$ for the FEM-simulation. The peak value given by the enhanced method shows a deviation of around $10 \%$, while the model using constant parameters calculates for currents more than $25 \%$ beyond the actual value of the FEM-model considering magnetic saturation. A similar effect shows in the comparison of the electric torque, where applying the enhanced model improves the deviations to FEM-simulation from $20.7 \%$ to $-7.1 \%$ in the peak values. 

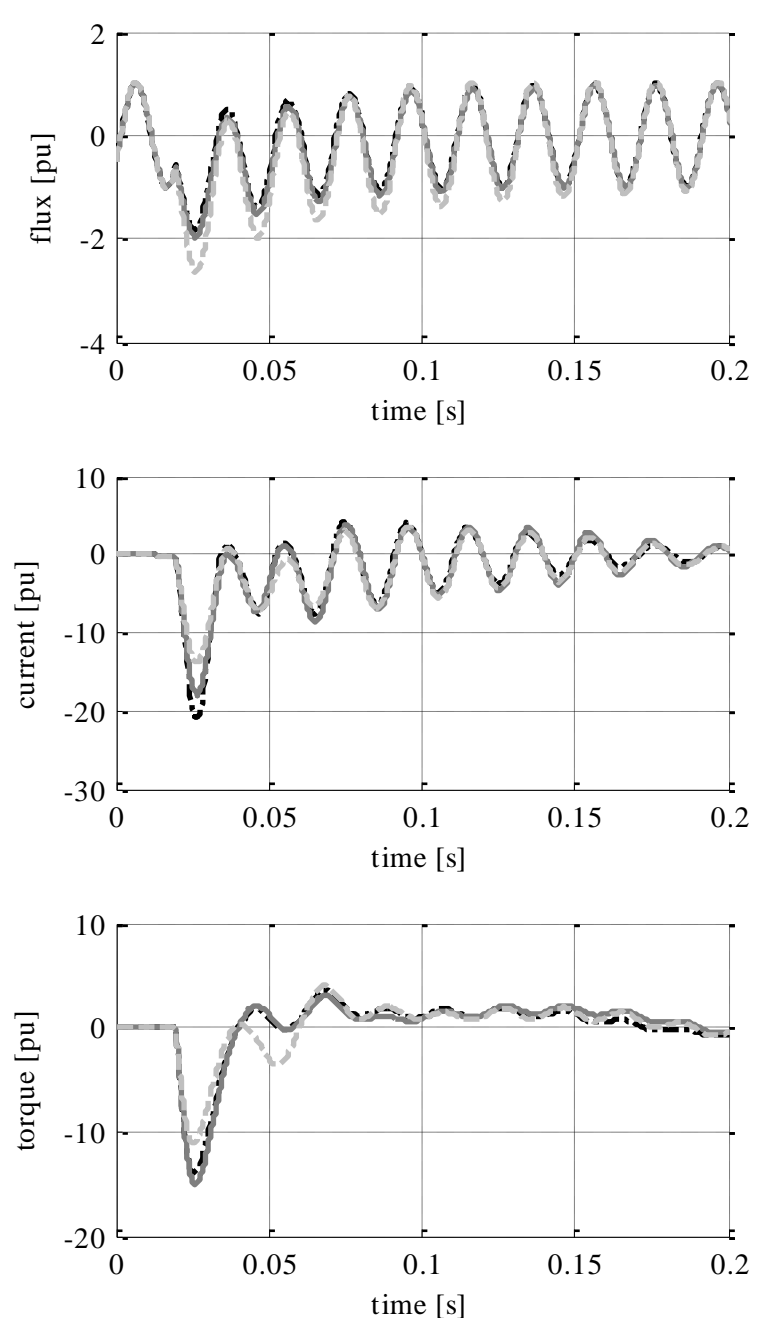

Figure 14: 180 degree out of phase synchronisation (black line -FEM, dark grey line -with saturation, light grey line -without saturation)

\section{SUMMARY AND PERSPECTIVES}

An investigation on the influence of magnetic saturation on the transient operating behavior of induction machines has been performed. For this purpose, inductances for an example motor have been calculated by using analytical approaches, assuming only magnetic voltage drop across the airgap and the machine slots, and static FEM, where non-linear magnetic behavior of the iron has been accounted for.

These results were subsequently converted into simplified current-depending parameter functions that can be used in transient machine models with a further enhanced space phasor approach based on the model of Tang [6]. In order to validate the results of the model, given by a set of differential equations which is similar to the symmetrical components with additional consideration of magnetic saturation in all flux paths of the Tequivalent circuit, a set of transients representing the most critical electrical and mechanical stress for the machine has been simulated using transient FEM.

The set consists of the startup from standstill, two- and threephase short circuit and out-of-phase synchronization with $180^{\circ}$ phase angle, with the further purpose of covering different phenomena with major influence on the machine transients, e.g. frequency of currents and torque, different mechanical speed, symmetrical- and nonsymmetrical behavior, highest peak currents, i.e. strongest saturation.

The results of the transients have been evaluated for the coupled flux and the current of the stator phases as well as for the electric torque produced. With the investigation purpose being on the comparison of saturated and non-saturated machine inductances, expectedly the most obvious deviation between the models (I - constant inductances, II - dynamically saturated inductances, III - FEM using non-linear magnetization curve) can be seen for the linked fluxes, where the enhanced model (II) shows very good accordance with the FEM throughout all transients investigated, whereas the simple model (I) shows obvious deviations at sequences with high ampere turns in the machine.

Even though the connection to saturation effects is less direct as for machine fluxes, a similar improvement can be observed in the accuracy of machine currents and the electric torque, where deviations at peak values between FEM and calculations based on differential equations are improved from values in the range of $10-25 \%$ (I) to values in the range of $2-10 \%$ (II).

The results obtained from the investigation clearly show that consideration of the non-linear magnetic behavior of the machine is inevitable in order to be able to precisely predict peak stresses and strains at machine transients. Even though the approach presented gives a complete outline from the determination of the current-dependent fluxes to application in an enhanced space-phasor model based on a system of differential equations, an interesting topic for further research will be to find fully analytical methods instead of using FEM to calculate the induction functions, so that FEM becomes obsolete not only for transient-, but also the static calculations giving the needed parameter functions.

\section{REFERENCES}

[1] P.D. Agarwal, P.L. Alger "Saturation Factors for Leakage Reactances", Power Apparatus and Systems, Part III. Transactions of the American Institute of Electrical Engineers, vol.79, no.3, pp.1037,1042, April 1960

[2] G. Angst, "Saturation Factors for Leakage Reactance of Induction Motors with Skewed Rotors", Power Apparatus and Systems, IEEE Transactions on , vol.82, no.68, pp.716,725, Oct. 1963

[3] C.A. Gross and R.M.Nelms, "A comparison of two analytical methods of determining the starting transients of a single phase induction machine," Electric Machines and Power Systems, Vol. 19, 1991, pp. 705-712.

[4] I.M. Nicolas, D. Demba, "Modeling and experimental study of 3-phase short-circuits of a double-cage induction machine," Electric Machines and Power Systems, Vol. 27, 1999, pp. 343-362.

[5] J. E Brown; K.P. Kovacs; P. Vas , "A Method of Including the Effects of Main Flux Path Saturation in the Generalized Equations of A.C. Machines," Power Apparatus and Systems, IEEE Transactions on , vol.PAS-102, no.1, pp.96,103, Jan. 1983

[6] Tang N.; Wu H.; Qiu P., "A saturation model of induction machine by space vector", Electrical Machines and Systems, 2001. ICEMS 2001. Proceedings of the Fifth International Conference on , vol.1, no., pp.85,88 vol.1, 2001

[7] G. Müller; K. Vogt; B. Ponick, "Berechnung elektrischer Maschinen“, Weinheim, Wiley-VCH, 2008

[8] P.C. Krause, "Anaysis of electric machinery“, New York, McGraw-Hill, 1987

[9] G. Müller; B. Ponick , "Theorie elektrischer Maschinen“, Weinheim, Wiley-VCH, 2009 


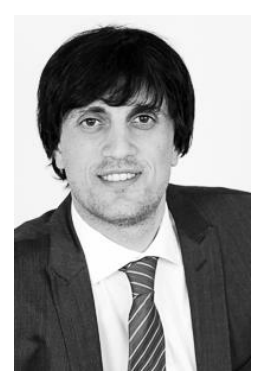

Alexander Conradi was born in BergischGladbach, Germany on August $30^{\text {th }}, 1981$. He studied Business Administration and Engineering at TU Dortmund University in Dortmund, Germany and for one year Chinese Language and Culture at Huazhong Shifan Daxue in Wuhan, China. He graduated in 2009. Since then, he is a scientific assistant at the Chair of Electrical Drives and Mechatronics of Prof. Dr.-Ing. Stefan Kulig. His main field of research is the dynamics of induction machines.

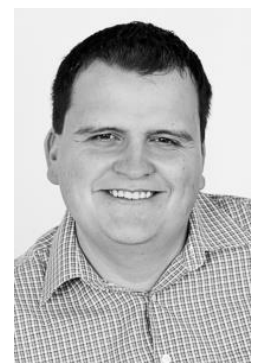

Christoph Schmülling was born in CastropRauxel, Germany on August $8^{\text {th }}$, 1986. He has studied at TU Dortmund University and received the degrees B.Sc. and M.Sc. in electrical engineering. In 2012, he joined the chair of Electrical Drives and Mechatronics of Prof. Dr.Ing. Stefan Kulig at TU Dortmund University as a research assistant. His main field of research is the dynamics of electric machines.

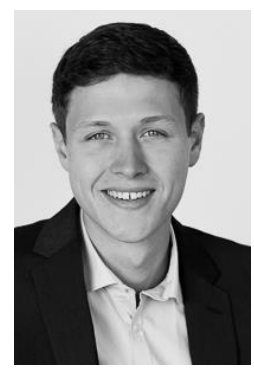

Stefan Schmülling was born in Herne, Germany on November $1^{\text {st }}, 1987$. He has studied at TU Dortmund University and received the degrees B.Sc. and M.Sc. in electrical engineering. In 2012, he joined the chair of Electrical Drives and Mechatronics of Prof. Dr.-Ing. Stefan Kulig at TU Dortmund University as a research assistant. His main field of research is the dynamics of electric machines. 